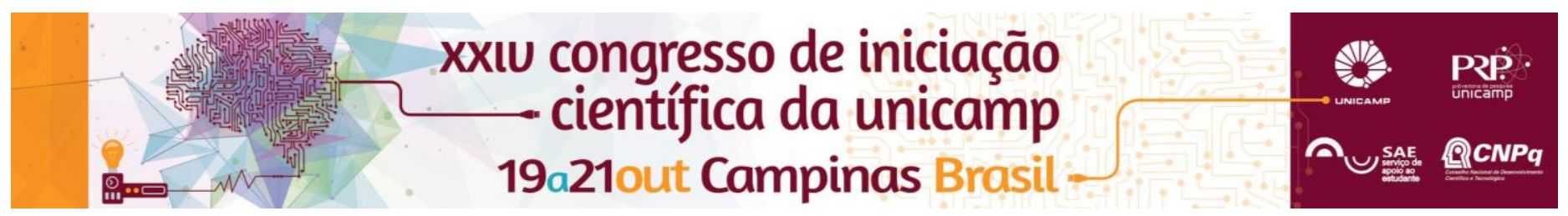

\title{
Content-Based Image Retrieval App using textures descriptors
}

\section{Alexandre Bastos Barrio* (IC)}

\section{Supervision: Prof. Léo Pini Magalhães, M.Sc. Agnus Azevedo Horta}

\section{Abstract}

This activity is part of a Doctoral Project whose objective is to perform a texture-based Content-Based Image Retrieval (CBIR) on Android Environment, allowing the user to choose an image input, his image database and the descriptor that will be used and, in addition, he will receive a feedback about its response. This project also focused on testing the viability to implement it on Android environment in terms of complexity and processing time.

Key words: CBIR, Descriptors, Android

\section{Introduction}

In order to retrieve an image from a database using another image as an input, different types of descriptors have been developed to extract the largest amount of information possible. This activity uses textures descriptors based on the LBP (Local Binary Pattern), an algorithm that analyses a single pixel per time and its surroundings to classify him.

The project was implemented using the Android environment in Android Studio IDE, allowing testing on portable devices compatible with this Operational System.

\section{Results and Discussion}

In the first stage, there were made theoretical studies about the LBP-based descriptors aiming to choose the ones that combine good accuracy and low processing time. After the implementation and tests phases, 3 algorithms were selected to be used in the app: Classic LBP ${ }^{1}{ }^{2}$, Uniform $\mathrm{LBP}^{4}$ and Fuzzy Uniform LBP 5 .

Initially, a prototype had been developed in order to implement these algorithms on Android environment. After obtaining good response, the final version of the product was created, with a simplified interface for the users.
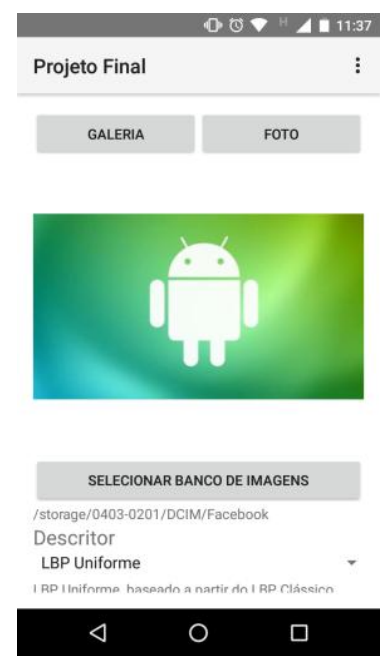

Image 1. Interface of the final product

During the development stage, the app was tested using an emulated device, a Nexus 5 Tablet 512 MB RAM, and also real gadgets: a Moto $G 2^{\text {nd }}$ gen. smartphone $1 \mathrm{~GB}$ RAM and a Samsung Galaxy Tab S 3GB RAM. The main image database used in most tests was the COIL-100, from Columbia University.

The major obstacle of the app is the time spent to extract the histogram from each image used and compared. The LBP algorithm is robust, but the processing time is affected by its complexity; and it gets worse when the database has numerous images with large dimensions.

\section{Conclusions}

Some changes were made to the code in order to reduce processing time and make possible the use of this app. The first modification was pre-calculate the histograms of images from the database, allowing the program to respond queries in a shorter time. Using the base COIL$100^{7}$, containing 7200 images, the time spent has shortened from more than 30 minutes to less than seconds. The second modification was to resize the images before extracting its features so that the decrease of the number of pixels reduced the processing time. This modification brings some loss of information, but they significantly minimizes the processing time.

These modifications were crucial to allow the viability of the app. Additionally, the tradeoff between precision and speed improved significantly its performance.

\section{Acknowledgement}

This work was supported by PIBIC (Scholarship Program for Scientific Support to undergraduate Students), a program from CNPq (National Counsel of Technological and Scientific Development) in partnership with Unicamp (State University of Campinas).

Also, I would like to thank Professor Léo Pini Magalhães and Agnus Azevedo Horta for their support.

${ }^{1}$ Wang, L., He, D.C., Texture Classification using Texture Spectrum, 1990 ${ }^{2}$ Ojala, T., Pietikainen, M., and Harwood, D., A comparative study of texture measures with classification based on featured distributions, 1996.

${ }^{3}$ Silva, A.T., Recuperação de imagens por conteúdo baseada em realimentação de relevância e classificador por floresta de caminhos ótimos, 2011.

${ }^{4}$ Nanni, L., Lumini A., Brahnam S., Survey on LBP based texture descriptors for image classification, 2012

${ }_{5}^{5}$ Ahonen, T., and Pietikainen, M., Soft Histograms for Local Binary Patterns, 2007.

${ }^{6}$ Iakovidis, D.K., Keramidas, E.G., and Maroulis, D.,Fuzzy Local Binary Patterns for Ultrasound Texture Characterization, 2008.

${ }^{7}$ S. A. Nene, S. K. Nayar and H. Murase, Technical Report CUCS-006-96, Columbia Object Image Library (COIL-100), 1996. 